\title{
«Tu sei la fiaba estrema»: le poesie di Alibi
}

\author{
Silvia CERACCHINI ${ }^{1}$ \\ Università degli Studi di Roma Tor Vergata \\ silvia.ceracchini@gmail.com
}

Recibido: $15 / 04 / 2013$

Aceptado: 20/06/2013

\section{RIASSUNTO}

Alibi, la prima raccolta poetica di Elsa Morante, esce nel 1958 per Longanesi. Le sue poesie risultano strettamente legate ai romanzi Menzogna e sortilegio e L'isola di Arturo, composti negli stessi anni, come l'autrice suggerisce nella Premessa, definendo i suoi versi come un «coro» dei suoi romanzi. Alla luce delle novità emerse dallo studio delle carte autografe dell'opera, depositate presso la Biblioteca Nazionale Centrale di Roma, vengono esaminati i versi di alcuni componimenti, e si propone un'analisi complessiva della raccolta, anche in relazione ai romanzi, secondo due grandi temi: i gatti, come simbolo dell'animalità immune dalla conoscenza del bene e del male, e la favola, con particolare riferimento alla teoria dell' "alibi», esposta in Sul romanzo, e a Sheherazade, la protagonista delle Mille e una notte che con le sue storie ha saputo sconfiggere la morte.

Parole chiave: Elsa Morante, Alibi, Poesie, i gatti, la favola.

\section{«Tu sei la fiaba estrema»: The Poems of Alibi}

\begin{abstract}
Elsa Morante's first volume of poetry, Alibi, was published in 1958 by Longanesi. Her poems are strongly linked to Menzogna e Sortilegio and L'isola di Arturo, novels composed in the same years. As stated by the author herself, the poems are a sort of «choir» to her novels. Due to the novelties emerged from the study of the original autographs of the texts, kept at the National Library in Rome, some verses are analyzed in view of the collection's relation to Morante's prose-fiction. An overall analysis of the volume is carried out according to two main themes: cats, symbols of instinctual animality, immune to the knowledge of good and evil; and the fable, with particular reference to the theory of the «alibi», formulated in Sul Romanzo, and to Sheherazade, the heroine of The Arabian Nights who defeats death with her tale-telling.
\end{abstract}

Keywords: Elsa Morante, Alibi, Poems, Cats, Fable.

SOMMARIO: 1 . Il corpus manoscritto 2. La raccolta 3. «DUE GATTI DI ELSA MORANTE» 4. La «camera di favole».

${ }^{1}$ Dipartimento di Italianistica, Facoltà di Lettere, Università degli Studi di Roma "Tor Vergata", Roma, Via Columbia 1, I-00133, Roma. 


\section{IL CORPUS MANOSCRITTO}

Le carte autografe di Alibi sono depositate presso la Biblioteca Nazionale Centrale di Roma all'interno del fondo A.R.C., recentemente catalogato da Giuliana Zagra e Leonardo Lattarulo in seguito a una seconda ingente donazione da parte degli eredi della scrittrice, Daniele Morante e Carlo Cecchi ${ }^{2}$. Come nel caso di altre opere edite, le progressive stesure manoscritte e dattiloscritte accolgono non solo le diverse versioni dei testi elaborate nel corso del tempo (con varie stratificazioni di varianti), ma anche appunti, note e promemoria, che costituiscono le linee guida del percorso compositivo. Le carte, tutti fogli sciolti, non sono ordinate in modo progressivo (almeno fino all'allestimento dei dattiloscritti, con numerazione autografa); non è quindi semplice orientarsi tra le numerose stesure. Il materiale è conservato in quattro cartelle, a loro volta raccolte da una cartellina di cartoncino di colore rosa, dal titolo Alibi $^{3}$, e seguito dalla nota (vergata con diversa penna e accompagnata da cancellature): «Brutte copie e manoscritti non definitivi di vari testi. Appunti presi durante il lavoro ecc. frammenti ecc.». La prima cartella contiene riscritture di diversi componimenti mentre la seconda accoglie solo le carte di Avventura, con titolo originario «Il cuore». Nella terza sono presenti principalmente le molte bozze di Alibi e nella quarta il dattiloscritto, con l'aggiunta di un indice manoscritto provvisorio. Le carte manoscritte sono prive di datazioni autografe a eccezione di una stesura di $A$ una bambina, datata «4 settembre 1945» (A.R.C. 52 I $4 / 1$ (1), c. $4 r$ ).

Per uno studio più completo sulla genesi della raccolta è necessario integrare a queste carte un importante quaderno, anch'esso catalogato recentemente nel fondo A.R.C., composto nella prima metà degli anni 40 (le datazioni autografe vanno dal 1943 al 1945), dal titolo Narciso. Versi poesie e altre cose molte delle quali rifiutate $^{4}$. Oltre ad altri versi, di grande interesse comparativo, confessioni personali, appunti e riscritture di parti di Menzogna e sortilegio, si trovano infatti appuntate nel quaderno le prove di alcune delle poesie di Alibi, risalenti a quegli

\footnotetext{
${ }^{2}$ Aggiungendosi al materiale donato nel 1989 e conservato nel fondo Vittorio Emanuele, che comprendeva i quaderni e le carte preparatorie dei principali lavori editi, i quattro romanzi e Il mondo salvato dai ragazzini (cfr. Zagra / Buttò 2006), il fondo A.R.C. ha arricchito un vero e proprio tesoro, colmando i vuoti di alcune opere edite, come appunto Alibi, e proponendo reperti importanti, primitive testimonianze di scrittura e interessante materiale inedito. In seguito alla costituzione del fondo è stata allestita una mostra che si è tenuta alla Biblioteca Nazionale Centrale di Roma (Santi, Sultani e Gran Capitani in camera mia. Inediti e ritrovati dall'Archivio di Elsa Morante. Biblioteca Nazionale Centrale di Roma, 26 ottobre 2012 - 31 gennaio 2013), corredata di un seminario di studi (20-21 novembre 2012) e di un volume curato da Giuliana Zagra. (Zagra 2012)

${ }^{3}$ Qui, come nelle altre citazioni, i sottolineati sono dell'originale. Dell'originale si conserva anche la differenza fra tondo e corsivo.

${ }^{4}$ Per una descrizione del quaderno si veda la scheda di Eleonora Cardinale in Zagra (2012: 93-102). Si rispetta la differenza fra tondo e corsivoo dell'originale.
} 
stessi anni: Minna la Siamese, Poesia per Saruzza, Amuleto, A una bambina, Sheherazade, Alla favola, Ai personaggi, Canto per il gatto Alvaro. La relazione è tale che il quaderno di Narciso può essere considerato come il primo nucleo di Alibi.

Nel caso di alcune poesie si riscontra una differenza di datazione tra le carte e la stampa. Poesia per Saruzza è datata nel libro «1943», mentre una sua bozza nel quaderno di Narciso ha data posteriore «25 marzo 1945» (A.R.C. 52 I 4/2 (1), c. 16r). Minna la Siamese distanzia temporalmente gli altri componimenti della raccolta con data «1941». In realtà all'interno delle carte sono presenti due diverse datazioni che riavvicinano la poesia alle altre degli anni 40: rispetto alla data della stampa, la bozza contenuta nel quaderno di Narciso è datata «giugno 1943» (A.R.C. 52 I 4/2 (1), c. 1r), mentre «1942» è la datazione del dattiloscritto (A.R.C. 52 I 4/2 (1) (4), cc. $3 r-4 r)$. È interessante al riguardo quanto dichiarato dall'autrice nella Premessa della raccolta: «Se, dunque, si è indotta a pubblicare questi versi (dei quali poi alcuni, come si vede dalle date poste in fondo, risalgono agli antichi tempi della sua prima giovinezza) 1'Autrice lo ha fatto soltanto nella speranza di rendere, a chi li leggerà, un poco di quel riposo, e divertimento, che lei stessa ne ha tratto al comporli»s ${ }^{5}$ (Morante 1988: 1373).

\section{LA RACCOLTA}

Uscita nel 1958 come secondo volume di una collezione di poesie per Longanesi ideata da Nico Naldini, insieme con Croce e delizia di Sandro Penna e L'Usignolo della Chiesa Cattolica di Pasolini, Alibi è la prima raccolta poetica di Elsa Morante. Il libro, ristampato con Garzanti nel 1988 e nel 1990 e con Einaudi nel 2004, copre un arco temporale di quasi vent'anni, accogliendo poesie composte tra i primi anni '40 e buona parte dei '50 e in parallelo con le stesure di Menzogna e sortilegio e dell'Isola di Arturo. La reciproca influenza tra i due romanzi e le poesie di Alibi è profonda e continua, tanto che si può intendere la raccolta come una sorta di controcanto poetico della prosa morantiana prodotta in questo ventennio di scrittura $^{6}$. La stretta relazione tra prosa e versi, testimoniata dall'importante ruolo di alcune poesie, poi accolte in Alibi, all'interno dei romanzi (Alla favola, Ai personaggi e Canto per il gatto Alvaro per Menzogna e sortilegio, L 'isola di Arturo

\footnotetext{
${ }^{5}$ In un'intervista a Schifano del 1984 Elsa Morante sottolinea la sua giovane età durante la composizione di Alibi: «Intanto le piacerebbe forse tradurre un libretto: "Alibi". Sono poesie. Ero giovane, allora...» (Schifano 1984: 127).

${ }^{6}$ Scrive Elena Porciani: «Nello stesso modo in cui nelle tragedie classiche il coro è spettatore interessato della vicenda che si svolge sulla scena, le poesie della Morante costituiscono nel loro insieme un autocommento alla propria narrativa» (Porciani 2006: 52).
} 
per l'omonimo romanzo, con titolo Dedica $)^{7}$ oltre che da una serie di ricorrenze, è ammessa dalla stessa Morante, che nella Premessa di Alibi specifica:

L'Autrice prega i lettori di perdonarle l'esiguo valore e peso di queste pagine. Essendo infatti, lei, per sua consuetudine (oltre che per sua natura e per suo destino) scrittrice di storie in prosa, i suoi radi versi sono, in parte, nient'altro che un'eco, o, se si voglia, un coro, dei suoi romanzi; e, in parte, nient'altro che un divertimento, o gioco, al quale essa ama talvolta abbandonarsi senza troppo impegno, per semplice piacere della musica. (Morante 1988: 1373)

A parte le poesie condivise, alcuni dati emersi dalle carte dimostrano come anche altri componimenti di Alibi siano in relazione con i romanzi ${ }^{8}$. Poesia per Saruzza (Morante 1988: 1377-1378), intitolata Eco nelle carte, presenta, invece della figura femminile della stampa «Nove anni da che t'ho salutata / o mia dimenticata, giovane siciliana», destinatario maschile: «Nove anni da che mi salutasti / o caro, fugace amante, giovane siciliano» ${ }^{9}$ (A.R.C. 52 I 4/2 (1), c. 15v). Diversi elementi farebbero pensare che l'originario protagonista maschile fosse ispirato al personaggio di Edoardo Cerentano. Oltre a una serie di coincidenze tematiche condivise tra la poesia e le pagine di Menzogna e sortilegio dedicate al lutto di Anna, come la separazione data dalla morte e la solitudine claustrofobica dell'io narrante ${ }^{10}$, specialmente il dato temporale dei nove anni, conservato nella stampa («Nove anni da che t'ho salutata»; «Ma l'eco d'una tua risata, / ultimo celeste addio / per nove anni si aggirò»), trova conferma nel romanzo: tra la morte del cugino e l'ultimo incontro con Anna, sono appunto trascorsi nove anni ${ }^{11}$. Elisa infatti, nata dal matrimonio tra Anna e Francesco, ha in quello stesso inverno «poco meno di nove anni» (Morante 1988: 579). Se per Poesia per Saruzza l'ispirazione del romanzo rimane solo un'ipotesi, il caso di Amuleto è più eloquente: la poesia è attestata nel quaderno di Narciso con titolo Ad un fantasma (A.R.C. 52 I 4/2 (1), c. 9r). Nei quaderni di Menzogna e sortilegio esistono informazioni precise riguardo

${ }^{7}$ La presenza delle poesie dei romanzi è segnalata da una nota editoriale. Riporto quella dell'edizione del 1958: «Le quattro poesie Alla favola, Ai personaggi, Canto per il gatto Alvaro e L'isola di Arturo (riprodotte nel presente volume per cortese concessione dell'editore Einaudi) fanno parte le prime tre del romanzo Menzogna e sortilegio e la quarta del romanzo L'isola di Arturo)» (Morante 1958: 82).

${ }^{8}$ Cfr. la scheda Alibi in Zagra (2012: 114-116).

${ }^{9}$ La bozza della poesia, contenuta nel quaderno di Narciso, è datata «25 marzo 1945».

${ }^{10}$ Cfr. i versi: «Fra noi due si distese / un'impervia rovina / di lontananza e tempo, / e il trombettiere delle morti / sui valichi suona il silenzio. [...] Poi, fu di nuovo il silenzio / nella memoria, / e io della vuota stanza / signora».

${ }^{11}$ «Eran trascorsi più di nove anni in simili vicende: e nell'inverno a cui siamo giunti con la nostra storia, già da due mesi Edoardo languiva, dopo l'ultima ricaduta del suo male. [...] Ma verso la fine del secondo mese, il professore la chiamò per comunicarle che la salute di Edoardo non si poteva più riconquistare con mezzi umani; e che, salvo un miracolo del cielo, prima che finisse l'anno Edoardo era perduto» (Morante 1988: 604-605). 
la collocazione della poesia, nata per entrare a far parte del romanzo. Nel quaderno XII si trova l'appunto: «Premettere all'inizio del libro Alla favola e dopo la chiusa A un fantasma» (V.E. 1619/xii, c. Iv). Nel piatto anteriore del quaderno XIV è inoltre presente lo schema d'inserimento delle poesie, con i primi versi di Alla favola («Di te, finzione...») e di quella che poi diventerà Amuleto («Quando tu passi...») ${ }^{12}$.

Salvo qualche sporadica reazione alla sua uscita, tra cui spicca l'articolo $L a$ grande pioggia di Giorgio Caproni (Caproni 1958), Alibi, vittima della subordinazione alle «storie in prosa» cui faceva coro, per lungo tempo è sopravvissuto per lo più inascoltato. Merito della sua riproposizione al pubblico e agli studiosi va a Cesare Garboli, curatore delle ristampe del libro, che per primo ne ammette la sottovalutazione:

Sono anch'io responsabile, come tanti altri, di scarso interesse e poca, pochissima attenzione nei confronti delle poesie di Elsa Morante. Responsabile come tanti altri di avere sottovalutato Alibi, di averlo penalizzato, di averlo gettato nello scaffale dopo un'occhiata distratta, o di averlo dimenticato sulla mensola vicino a dove si passa sempre e non si guarda mai. [...] Il fuoco era di paglia. Tutti si chetarono. Alibi fu dimenticato. (Garboli 2004: vii)

La ristampa del 2004 in particolare, rispetto alle precedenti, ha la novità di includere in appendice le tre strofe di una poesia inedita, dal titolo Narciso, trovata all'interno del quaderno di Narciso. In aggiunta alle tre strofe pubblicate da Garboli sono state recentemente da me ritrovate due strofe di conclusione della poesia, presentate nel corso della giornata di studi della Biblioteca Nazionale Centrale di Roma (20-21 novembre 2012), correlata alla già citata mostra Santi, Sultani e Gran Capitani in camera mia. Inediti e ritrovati dall'Archivio di Elsa Morante.

L'autrice in prima persona dichiara nelle interviste di scrivere poesie «nei momenti di felicità», in quanto «modo d'esprimere gioia» (Barbato 1963: 3), e di trovare nella scrittura in versi ristoro dalla faticosa scrittura dei romanzi ${ }^{13}$. Sebbene alcuni componimenti, leggeri e disimpegnati, composti da immagini semplici e quotidiane, confermino questa impressione generale di leggerezza e semplicità (si pensi ad esempio a Minna la Siamese), altri non la condividono e tendono invece a versi complessi e introspettivi, dai toni più cupi (si pensi ad esempio ad Avventura). La laboriosa storia manoscritta di alcune poesie smentisce inoltre lo scarso impegno dichiarato nella Premessa («i suoi radi versi sono, in parte [...] nient'altro che un divertimento, o gioco, al quale essa ama talvolta abbandonarsi senza troppo impegno, per semplice piacere della musica»), confermando al contrario la dedizione riservata alla composizione in versi. Non è un caso che in entrambi i

${ }^{12}$ In particolare la poesia doveva essere collocata in apertura della Parte VI: «(Anna) (Levati, o pernice dei morti)».

13 "“Cercare il ritmo, la parola che fa poesia" - dice - "è più riposante di tutto"» (Massari 1957: 15) 
romanzi Menzogna e sortilegio e L'isola di Arturo, le poesie non abbiano ruolo esornativo bensì esplicativo, esistendo come veri e propri fulcri della narrazione. In molte interviste inoltre la Morante, che nella Premessa si definisce «per sua consuetudine (oltre che per sua natura e per suo destino) scrittrice di storie in prosa», ammette di amare particolarmente la sua poesia. In un'intervista del 1984 afferma: «Veramente il mio libro prediletto è Il mondo salvato dai ragazzini. C'è un altro mio libro che amo ed è un libro di poesie, Alibi» (Poggio 1984: 38) ${ }^{14}$. Già nell'intervista a Saviane del 1955 veniva dichiarato l'amore per questo primo libro di poesie: «Elsa Morante non scrive articoli per giornali. Preferisce dedicarsi ai suoi libri e alle sue poesie. Ha finito un'opera insolita nella nostra letteratura. È una composizione in versi, Alibi, "quasi un poema", lei dice, cui è molto affezionata» (Saviane 1955: 11).

Dalle poesie di Alibi emerge chiaramente una ricerca d'amore costante e mai appagata, sintomo di un bisogno profondo e intimo, che contraddistingue in fondo l'intero universo poetico morantiano. La tematica è infatti ben riscontrabile nel titolo pensato per il quaderno di Narciso. Poesie d'amore di Narciso a se stesso, le cui alternative Finzione e Il Sosia pure suggeriscono il mito di Narciso come immagine di nascita del libro. La storia di Eco e Narciso si accorda perfettamente con l'analisi di Cesare Garboli riguardo ad Alibi, in cui il critico riconosce una «capacità di amare senza risparmio, una capacità insolita, e quasi mostruosa, di regalarsi all'amore» (Garboli 2004: ix). Lo stesso titolo definitivo, Alibi, se pensato in relazione ai precedenti titoli per il quaderno di Narciso, si arricchisce della creazione di un nesso ossimorico in cui l'io appare sdoppiarsi in due entità distinte ma segretamente coincidenti, come conferma l'impiego del termine nell'articolo Sul romanzo del 1959 (vd. infra), per spiegare l'utilizzo della prima persona come punto di vista del romanzo:

E allora, nel momento di fissare la propria verità attraverso una sua attenzione al mondo reale, il romanziere moderno, in luogo di invocare le Muse, è indotto a suscitare un io recitante (protagonista e interprete) che gli valga da alibi. Quasi per significare, a propria difesa: «S'intende che quella da me rappresentata non è la realtà; ma una realtà relativa all'io di me stesso, o ad un altro io, diverso in apparenza, da me stesso, che in sostanza, però, m'appartiene, e nel quale io, adesso, m'impersono per intero». (Morante 1990: 1505)

Alcuni studiosi hanno indicato, come ispiratori della raccolta, i nomi di Leopardi, Saba, Penna, a cui è dedicata la poesia scherzo Il gatto all'uccellino, e Montale (Venturi 1977: 86). Il ricordo di questi autori canonici è facilmente percepibile dalla lettura dei versi, grazie alla ricorrenza d'immagini e termini propri

14 Anche nell'intervista a Costantini la Morante conferma di considerare il suo libro prediletto Il mondo salvato dai ragazzini: «Il mondo salvato dai ragazzini è il libro che preferisco, è il mio libro migliore, è il più bello dei miei libri, forse è il solo libro che abbia scritto. Se dovessi portarmi uno dei miei libri nell'altro mondo, non esiterei un momento, mi porterei Il mondo salvato dai ragazzini.» (Costantini 1980) 
di questi grandi poeti amati dalla Morante. Basti pensare ad esempio alla poesia $A$ una bambina (Morante 1988: 1380): «Sembrano i tuoi capelli la lustra piuma / d'un nero anatretto. Gli occhi / simili a foglie screziate. Semi d'oro tu hai / sulle guance: i tuoi pallori / aman l'ombra». La poesia, composta da versi di sabiana memoria, incentrata sul foro inutilizzato per gli orecchini, oggetto che rievoca Montale insieme alla «vasca iridescente» ${ }^{15}$, si nutre della tradizione precedente, pregiandosi dell'aggettivo leopardiano «solinga» («Ma tu solinga stai, dei curiosi / nulla t'importa»). Anche l'importanza conferita ai diversi animali presenti nella raccolta risente della lezione petrarchesca e soprattutto leopardiana, chiamata direttamente in causa da quel ripetuto «spaùro» che, in Minna la Siamese (Morante 1988: 1375), riassume tutta la distanza tra l'uomo e l'animale: «Se penso a quanto di secoli e cose noi due divide, / spaúro. Per me spaúro». L'influenza di Leopardi, lettura specialmente dei tempi giovanili ${ }^{16}$, si percepisce poi in particolare nella chiusa di Alla favola (Morante 1988: 1384), «la risposta celeste / mi fingo», come pure nei versi di Su Nerina (Morante 1988: 1396), che già dal titolo sembrerebbe riprendere non casualmente la Nerina delle Ricordanze. Primo elemento comune tra i due componimenti, oltre al nome della figura femminile, è il tema del recupero memoriale. Su Nerina si apre infatti con: «Ricordo d'una infanzia». La vitalità e lo scenario di festa della poesia, fotografato nel particolare dell'abito della protagonista ( $($ Festante metropoli terrestre, e tu, Laude, Gerusalemme / dei mattini domenicali! / Lei, nel vestito nuovo, / pari alla regina di Saba...») contrasta con un finale sospeso, allusivo e funebre: «Troppo lunga l'attesa del giorno. / Si lasciò tentare dal sonno» ${ }^{17}$. Stesso contrasto si ha nell'ultima strofa delle Ricordanze. Di

15 Si pensi al componimento di Ossi di seppia, Vasca. Anche l'aggettivo «screziate» («Gli occhi / simili a foglie screziate») richiama il Montale di Dora Markus: «E qui dove un'antica vita / si screzia in una dolce / ansietà d'Oriente, / le tue parole iridavano come le scaglie / della triglia moribonda». La lettura di Montale negli anni 40 è testimoniata dai quaderni di Menzogna e sortilegio, dove la Morante ha appuntato suoi numerosi versi. Nel quaderno XXII, in data «27 aprile 1946», è presente ad esempio per intero Molti anni, e uno di più duro sopra il lago, poesia in cui è per altro nominato San Giorgio, come anche in Avventura.

${ }^{16}$ In un'intervista a Lino Del Fra per L'Italia che scrive: «Elsa Morante accenna ai suoi esordi letterari, ai tempi della prima gioventù, allorché le sue preferenze letterarie si chiamavano Kafka e Leopardi: "Da ragazzi, proprio perché si è molto ottimisti, ci si sente irresistibilmente attratti dal pessimismo. Ragazzetta, scrivevo durante la notte e mi riposavo al mattino"» (Del Fra 1957: 135). In Aracoeli, l'ultimo romanzo morantiano, Manuele porta nel suo zaino i suoi oggetti più cari, tra cui i Canti di Giacomo Leopardi: «E io le posai dinanzi, sul tavolino del Caffè, l'unico bagaglio che m'ero portato nella fuga [...]. Conteneva l'astuccio degli occhiali, un ciuffo di peli del mio cane Balletto, e i Canti di Giacomo Leopardi.» (Morante 1990: 1439)

${ }^{17}$ Ricordo che Nerina è il titolo di un romanzo mai completato che con L'isola di Arturo avrebbe dovuto creare un dittico dal titolo Due amori impossibili, come risulta da un'intervista a L'Unità rilasciata il 24 marzo 1952. Il romanzo avrebbe trattato «di una 
Nerina, simbolo della vita giovane e festosa, non è rimasta che la «rimembranza acerba»: «Se a feste anco talvolta, / se a radunanze io movo, infra me stesso / dico: o Nerina, a radunanze, a feste / tu non ti acconci più, tu più non movi». In $S u$ Nerina si intreccia con la suggestione leopardiana una poesia di Arthur Rimbaud, tratta da Illuminations, intitolata Enfance. In una bozza della poesia (A.R.C. 52 I 4/1 (1), c. 14r) è presente infatti la nota autografa: «Credo superfluo avvertire i miei lettori che le frasi in corsivo sono tradotte da Enfance di Rimbaud e che tutte queste strofe sono quasi delle variazioni intorno a frasi del poema suddetto». Accantonato il progetto iniziale di costruire Su Nerina sulle traduzioni di Enfance, dei versi tradotti presenti nelle diverse bozze della poesia ${ }^{18}$ rimane vaga e impercettibile traccia, oltre che nel riferimento al titolo nell'incipit «Ricordo d'una infanzia», nei versi «Senza genitori né avi» (da «sans parents ni cour») e «archi e colonne d'oro», da «L'essaim des feuilles d'or», che nei manoscritti era ricalcato più esplicitamente sul modello francese: «lo sciame delle foglie d'oro». Il verso di Enfance «Des bêtes d'une élégance fabuleuse circulaient», inserito tra le traduzioni della bozza di $\mathrm{Su}$ Nerina, è appuntato, questa volta in francese, anche in una bozza del Canto per il gatto Alvaro (A.R.C. 52 I 4/2 (1), c. 10r), in data «15 dicembre 1944», e tornerà infine, tradotto ed evidenziato dal maiuscolo, nel componimento del Mondo salvato dai ragazzini, La smania dello scandalo: «LE BESTIE D'UNA ELEGANZA FAVOLOSA» (Morante 1990: 130). Il verso, evidentemente caro alla Morante, è presente inoltre, nella forma «C'erano le belve d'eleganza favolosa», insieme con la traduzione «lo sciame delle foglie d'oro», in una poesia dedicata a Luchino Visconti, pubblicata nell'epistolario L'amata. Lettere di e a Elsa Morante, uscito per Einaudi nel 2012 a cura di Daniele Morante con la collaborazione di Giuliana Zagra. (Morante 2012: 313-314)

L'influenza di Rimbaud, pur se ridimensionata nella stampa, è il segno evidente dell'apertura della raccolta verso letterature diverse, confermata anche dall'appunto autografo tratto da The pains of sleep di Coleridge «and whom I scorned, those only strong!» (A.R.C. 52 I 4/2 (1), c. 16r), e dalla citazione dal titolo del romanzo di

fanciulla di un minatore, che ama appassionatamente la danza, e che muore mentre sta per realizzare il suo sogno.» (Cecchi / Garboli 1988: LIX)

${ }^{18}$ Le traduzioni, sottolineate, si modificano nel corso delle riscritture. Riporto solo le versioni presenti alle cc. $14 r-15 r$ di A.R.C. 52 I 4/1 (1): «Senza parenti né corte» («sans parents ni cour»), «lo sciame delle foglie d'oro» («L'essaim des feuilles d'or»), «s'aggiravano bestie d'una eleganza favolosa» («Des bêtes d'une élégance fabuleuse circulaient»), «La giovane madre defunta scende dalla loggia» («La jeune maman trépassée descend la perron»), «Flutti senza navi, nudità rivestite dagli arcobaleni!» («nudité qu'ombrent, traversent et habillent les arcs-en-ciel, la flore, la mer»), "Così alte le palizzate che si vedon solo le cime oscillanti. Del resto, là dentro non c'è nulla da vedere» («Les palissades sont si hautes qu'on ne voit que les cimes bruissantes. D'ailleurs il n'y a rien à voir là-dedans»)). 
Truman Capote «altre voci altre stanze» in Amleto ${ }^{19}$ (Morante 1988: 1400), perfettamente in linea con la fenomenologia dell'alibi.

Oltre alle letture citate, alcuni elementi della raccolta sembrano suggerire la suggestione di Francesco Petrarca. In particolare è interessante porre a confronto il sonetto proemiale del Canzoniere, Voi ch'ascoltate in rime sparse il suono, e la già citata Premessa di Alibi. Nonostante la lunga composizione dell'opera, testimoniata dalle numerose carte autografe, e il ruolo riservato non solo ai componimenti presenti all'interno dei primi due romanzi, ma anche agli altri, che della prosa costituiscono un «coro» - come la stessa Premessa suggerisce - («i suoi radi versi sono, in parte, nient'altro che un'eco, o, se si voglia, un coro, dei suoi romanzi»), ma in una relazione tutt'altro che impari, come nel teatro greco, la scrittrice tratta $i$ suoi versi in termini di nugae $e^{20}$, e si rivolge al lettore per chiedere perdono («L'Autrice prega i lettori di perdonarle») per «l'esiguo valore e peso di queste pagine» che sta per leggere. Anche Petrarca, che alle sue «rime sparse» in volgare ha dedicato, come la Morante, molta più cura e dedizione di quanto non dichiari, invocando il lettore in prima persona nel sonetto, ammette di cercare «perdono». La stessa esplicitazione del dato temporale («dei quali alcuni, come si vede dalle date poste in fondo, risalgono agli antichi tempi della sua prima giovinezza»), trova riscontro nell'indicazione temporale riguardo il «giovenile errore» della premessa petrarchesca. Pur se in toni e con intenzioni ovviamente diverse, quasi parodiche nel caso morantiano, le due premesse sembrano dunque condividere diversi temi, alcuni dei quali espressi di nuovo nel Congedo del Mondo salvato dai ragazzini, in una ripresa questa volta palesemente esplicita e quasi letterale del primo sonetto del Canzoniere: «E adesso, o voi che avete ascoltato queste canzoni, / perdonatemi se sospiro ripensando / a quanto era stata semplice / la mia vita» (Morante 1990: $249)^{21}$. In $A i$ personaggi inoltre, la poesia di Menzogna e sortilegio che spiega il meccanismo della narrazione e la mansione di Elisa rispetto ai fantasmi di cui racconta la storia, la Morante riprende l'immagine cara a Petrarca, già presente in Seneca e Orazio, del poeta che crea nuova materia artistica traendo ispirazione dalla tradizione, come le api producono il miele dal contatto con più fiori: «Altro io non sono che pronuba ape / fra voi, fiori straordinari e occulti. / Ma sulle effimere mie elitre / pur vaga una traccia rimane / del vostro polline celeste. / E il vostro miele / è tutto mio!» (Morante 1988: 1385). Se considerata dal punto di vista più generale della scrittrice o indipendentemente dalla sua collocazione all'interno del romanzo, la poesia può essere intesa come apertura, da parte di Elsa Morante, verso la

${ }^{19}$ In una lettera a Tommaso Landolfi, riportata nell'Amata, la Morante, a proposito di «altre voci altre stanze», commenta: «è un bel titolo.» (Morante 2012: 114)

${ }^{20}$ Si pensi a come i «radi versi» della Premessa ricordino le «rime sparse» del sonetto di Petrarca.

${ }^{21}$ Sulla ripresa del sonetto petrarchesco nel Congedo cfr. Bardini (1999: 619). Cfr. inoltre il lavoro di Maurizio Fiorilla (2009: 257-258) su Addio che ha messo in luce come l'elaborazione del Congedo sia stata condizionata dal desiderio di avvicinarsi al modello petrarchesco. 
tradizione letteraria italiana e internazionale, come la predisposizione a un dialogo continuo e proficuo con $\mathrm{i}$ «miniati volumi» della sua stanza.

\section{3. «DUE GATTI DI ELSA MORANTE»}

Rispetto alle diverse tematiche che attraversano i componimenti di Alibi, si è scelto di analizzarne due in modo particolare: il mondo animale, rappresentato soprattutto dai gatti, e la fascinazione della favola.

Caratterizzato da versi semplici e da un andamento melodico e lineare, il primo componimento della raccolta, Minna la Siamese (Morante 1988: 1375), ha per oggetto una siamese di nome Minna, identificata nel suo essere animale prima che gatto: «Ho una bestiola, una gatta: il suo nome è Minna». La poesia indaga la bestialità di Minna come condizione perenne d'inconsapevolezza: «Se penso a quanto di secoli e cose noi due divide, / spaúro. Per me spaúro: ch'essa di ciò nulla sa». Nell'articolo Il paradiso terrestre. Il vero re degli animali, pubblicato il 30 dicembre 1950 su «Il Mondo» e ora in Pro o contro la bomba atomica (Morante 1990: 1475-1477), la differenza tra uomo e animale viene spiegata proprio in ragione della loro diversa «conoscenza del bene e del male»:

Come tutti sanno, nel gustare questo frutto, l'uomo acquistò la conoscenza del bene e del male, vale a dire la capacità di giudizio. Ma gli altri animali rimasero immuni da simile capacità: è questo il carattere più amabile che distingue gli altri animali dall'uomo; ed è qui che risiede soprattutto la grazia della loro compagnia. (Morante 1990: 1475)

L'essenzialità dei versi di Minna la Siamese si accorda con la descrizione dei movimenti felini, espressione di una semplicità primordiale incapace di giudizio, che è «il carattere più amabile che distingue gli altri animali dall'uomo». L'illusione di poter trovare un ponte tra i due mondi, racchiusa nelle immagini del tremore dell'orecchio della gatta al sentir pronunciare il suo nome e della «chitarretta» ch'essa ha per esprimere gioia o gratitudine ${ }^{22}$, svanisce nella chiusura:

Tanto mi bacia, a volte, che d'esserle cara io m'illudo, ma so che un'altra padrona, o me, per lei fa uguale.

Mi segue, sì da illudermi che tutto io sia per lei,

ma so che la mia morte non potrebbe sfiorarla...

In questi versi l'espressione di una coscienza disincantata e prettamente umana, consapevole della caducità della vita e dello scorrere del tempo, non trova affermazione diretta ma riflessa, tramite la rappresentazione della straordinaria

22 «Sulle ginocchia mi viene, mi guarda, e poi dorme, / tale che mi dimentico d'averla. Ma se poi, / memore, a nome la chiamo, nel sonno un orecchio / le trema: ombrato dal suo nome è il suo sonno. // Gioie per dire, e grazie, una chitarretta essa ha: / se la testina le gratto, o il collo, dolce suona». 
incoscienza animale di Minna. Il confronto con l'animale, di rimembranza leopardiana, come pure il già citato verbo "spaùro», è però qui solo suggerito, a differenza dell'uso caro a Leopardi (si pensi ad esempio al Passero solitario o al Canto notturno di un pastore errante dell'Asia e all'esplicitarsi del paragone): l'attenzione rimane focalizzata sulla gatta Minna, senza mai spostarsi direttamente sul sentimento umano, sollecitato solo attraverso l'animale. Ciò è particolarmente evidente nel verso finale, dove l'elemento della morte che emerge improvviso è espresso indirettamente, con incisivo impatto, attraverso il punto di vista di Minna, simbolo d'inconsapevolezza: «ma so che la mia morte non potrebbe sfiorarla...». L'intenzione di chiudere la poesia con un colpo inaspettato, che si ponesse in controtendenza rispetto ai versi precedenti, potrebbe aver condizionato la decisione di porre la stanza, che aveva in precedenza posizione interna, come ultima. Il tono della stanza attestata come ultima nel dattiloscritto era infatti decisamente diverso, privo della sferzata finale (A.R.C. 52 I 4/1 (4), c. $4 r$ ):

Se, dietro i vetri, sul tetto un passerotto scorge,

gli orecchi leva, e scuote la coda: quasi pare

festa o stupore farsi di lui, che vola piumato,

ma pensa: ah, dolce cosa conquistarlo, e mangiarselo!

L'eliminazione dei versi, come già notato da Marco Bardini (1999: 174-187), potrebbe inoltre aver coinciso con la composizione della poesia Il gatto all'uccellino, che di questa stanza condivide tema e stile (Bardini 1999: 177-178). Con la definitiva chiusura la poesia nega la possibilità di riportare il tono alla leggerezza delle strofe iniziali, contrastando così con queste in modo netto e irrecuperabile. La distanza abissale tra i due mondi, quello umano e quello felino, trova ancora conferma nell'articolo Il paradiso terrestre. Il vero re degli animali, nella teorizzazione morantiana del gatto, e in particolare del siamese, come il maggiore tra gli animali, «questo circo angelico», nel quale è conservato un ricordo dell'«Eden perduto»:

Lodiamo tutta la multiforme nazione dei nostri compagni animali, questo circo angelico in cui l'uomo può riconoscere, a testimonianza del suo rango perduto, la nobile infanzia dell'Eden. Ma, fra tutti gli animali esistenti, il primato assoluto lo decretiamo al gatto siamese $^{23}$.

${ }^{23}$ Dalle pagine di diario composte nell'estate del 1952 e pubblicate postume nel febbraio 1988 su Paragone Letteratura: «Il gatto Giuseppe è morto il $1^{\circ}$ Agosto. Era il mio più caro amico, la metà della mia anima. I suoi occhi erano gli occhi più meravigliosi che mai mi siano apparsi. Mi è impossibile credere che si siano spenti per sempre. Quali occhi, umani o non umani, ebbero mai per me quella luce di Paradiso, e quello straordinario affetto, quella partecipazione a tutti i sentimenti del mio cuore, non detti e indicibili! [...] Posso dire che, fino ad oggi, io sono arrivata a conquistare una sola verità assoluta: gli animali sono gli angeli. E fra questi angeli: gli arcangeli, le fate, sono i gatti siamesi» (Cecchi / Garboli 1988:1x). 
L'articolo conclude con l'investitura di «vero re degli animali» del gatto siamese, la cui totale incoscienza è garanzia di umiltà assoluta:

Quanto a noi, fra tanto mutare di regni e di corone, osiamo qui proporre la candidatura del gatto siamese a vero re degli animali. Siamo certi che questo titolo non gli farà perdere la sua nativa discrezione e affabilità. Infatti, non avendo mangiato il frutto della scienza del bene e del male, Egli fa minor conto del titolo di re che d'un pesciolino; e non potrà mai montarsi la testa.

La connessione tra l'articolo e la poesia è confermata dalla ricorrenza del «pesciolino», dato a Minna nei giorni di festa: «Perché celebri anch'essa, a pranzo le do un pesciolino; né la causa essa intende: pur beata lo mangia».

L'indicazione della razza aiuta a giustificare l'evoluzione del titolo Minna la Siamese: la specificazione «la siamese» è aggiunta dall'autrice a penna solo a partire da una delle versioni dattiloscritte (A.R.C. 52 I 4/1 (4), c. 55r), accanto al più generico «Minna», attestato nelle versioni dattiloscritte presumibilmente precedenti e nel manoscritto contenuto nel quaderno di Narciso. Il particolare statuto del gatto siamese, il «vero re degli animali»e fra tutti il più «angelico», trova riscontro nella descrizione di Minna. Oltre ad una certa umile regalità che la contraddistingue, la siamese sembra avere, con quelle sue «iridi celesti», aggettivo dalla connotazione non solo cromatica, la grazia delle donne stilnoviste, inconsapevoli di portare in terra un miracolo del cielo. Forse non è casuale la suggestione del verso «ma lei, tanto è gentile, sol per gioco li adopra», dove riecheggia il sonetto dantesco Tanto gentile e tanto onesta pare.

L'universo animale, e soprattutto felino, è di grande importanza per Alibi, tanto che «Minna» (non ancora «la Siamese») doveva essere inserita, secondo le indicazioni del dattiloscritto, in una sezione intitolata «DUE GATTI», con sotto la specifica «di ELSA MORANTE» (A.R.C. 52 I 4/1 (4), cc. 27r-28r), insieme con il Canto per il gatto Alvaro, la poesia di chiusura di Menzogna e sortilegio ${ }^{24}$. Presentato inizialmente nel romanzo come figura misteriosa, «creatura vivente, sí, ma non umana» (Morante 1988: 17), il gatto Alvaro è «un personaggio dei più amabili e importanti» (Morante 1988: 942), l'elemento vitale del libro, l'unica consolazione e compagnia di Elisa nel coro dei suoi morti ${ }^{25}$ :

Terzo, e sopra tutti amato, è il qui presente Alvaro, solo mio compagno vivente in questa camera solitaria. Com'io m'accingo a tracciare qui sotto la parola fine, egli che m'è stato sempre vicino mentr'io scrivevo questa lunga storia, mi guarda coi suoi graziosi occhi fedeli. E sembra dire a Elisa che, nonostante tutto, l'innocenza e l'amicizia dureranno finché duri il mondo. (Morante 1988: 941)

${ }^{24}$ Sul Canto per il gatto Alvaro e le altre poesie di Menzogna e sortilegio, cfr. Bardini (1999: 292-302) e Rosa (1995: 22-26).

${ }^{25}$ Uno dei titoli presenti nel quaderno di Narciso è infatti «Fantasmi e gatti / Poesie». 
La fedele compagnia di Alvaro, manifestazione di un amore incondizionato e scevro da narcisismi, al contrario di quello tra umani, è anticipata dal «solitario gatto della bottega» (Morante 1988: 613) con cui Elisa divide il suo «panino», giudicandolo il suo «più amato passatempo», e dal «gattino di rara bellezza» incontrato da Elisa in sogno. Nonostante la paura di essere rifiutata per via della pelle scura che la bambina sogna di avere, Elisa trova nel gattino un'offerta d'affetto senza riserve e incurante della sua presunta diversità: «Ma il gattino si strusciò contro le mie guance, miagolando così dolcemente da parere, piuttosto che un micio, un usignolo» (Morante 1988: 744-745). Anche nell'articolo Il paradiso terrestre. Il vero re degli animali è sottolineata la straordinaria virtù dell'animale di non distinguere tra le qualità umane, perché «la bruttezza, la malattia e la vecchiaia non sono vizi ripugnanti per lui» (Morante 1990: 1476). Al pari di Minna la Siamese anche il Canto per il gatto Alvaro (Morante 1988: 1386-1387) è in stretto contatto con i concetti espressi nell'articolo. Privo di qualsiasi consapevolezza, Alvaro, prova evidente dell'esistenza di un Eden celeste di cui anche l'uomo aveva potuto godere prima della sua cacciata, è un beato abitante del «Paradiso» da cui Elisa è ormai esiliata, avendo mangiato il frutto amaro della conoscenza:

E t'ero uguale!

Uguale! Ricordi, tu, arrogante mestizia? Di foglie tetro e sfolgorante, un giardino abitammo insieme, fra il popolo barbaro del Paradiso. Fu per me l'esilio, ma la camera tua là rimane, e nella mia terrestre fugace passi giocante pellegrino.

La posizione della poesia all'interno del romanzo, di grande rilievo, costituisce una sostanziale inserzione della vita e della speranza («E sembra dire a Elisa che, nonostante tutto, l'innocenza e l'amicizia dureranno finché duri il mondo») in un contesto di morte e desolazione. Tuttavia, come nel caso di Minna la Siamese, la presenza del gatto, che pone in contatto due mondi tangibili ma di fatto lontanissimi ( «Fu per me l'esilio, / ma la camera tua là rimane, / e nella mia terrestre fugace passi / giocante pellegrino»), è anche contemporaneamente un'amplificazione, per contrasto, della diversità e della solitudine di Elisa ${ }^{26}$; Alvaro ne rappresenta il doppio («E t'ero uguale!»), il suo unico vero amico («L'allegria d'averti amico / basta al cuore») e condivide con lei, nel suo essere gatto, la capacità di vedere $\mathrm{i}$

26 Introducendo «il misterioso Alvaro» (Morante 1988: 16), Elisa distanzia la sfera umana da quella animale: «Ma siccome, per gli uomini, la compagnia d'un Alvaro non conta, io sono, in breve, sola.» (Morante 1988: 18) 
fantasmi ${ }^{27}$, ma ne identifica una condizione primitiva e irraggiungibile: rispetto ai connotati di «perenne», «libero» e «ingenuo» del gatto Alvaro, Elisa o più generalmente l'io poetico, al contrario degli «animali celesti», «tre cose» ha «in sorte», «prigione peccato e morte». Nonostante la compagnia del gatto sia un'indiscussa consolazione ( $« \mathrm{E}$ di mie fole e stragi / coi tuoi baci, coi tuoi dolci lamenti, / tu mi consoli, / o gatto mio!» $)^{28}$ e un assaggio di Paradiso terrestre, la sua natura straordinaria marca al tempo stesso l'estraneità e l'esilio del mondo umano. Elisa, consapevole di appartenere alla categoria umana, accetta l'amore del suo angelico amico come un mistero inconoscibile («Perché mi concedi / il tuo favore, o selvaggio?»; «Segreti di fiere / non si dicono a donne»). Lo scambio tra i due non è di pari livello («Non mi rispondi?»), ma ha il tono mistico della confessione e della preghiera: «Le confidenze invidiate / imprigioni tu, come spada di Damasco le storie d'oro / in velluto zebrato». Il gatto Alvaro è una consolazione e una lusinga («Chiudi gli occhi e cantami / lusinghe lusinghe coi tuoi sospiri ronzanti»), ed è la vita che s'impone nella camera della «sepolta viva» Elisa, ma è anche la raffigurazione della nostalgia dell'incoscienza e di un Eden celeste perduto che è rimasto nei suoi occhi come in quelli di Minna.

Quella che sembrerebbe essere la prima bozza della poesia, contenuta nel quaderno di Narciso, con data «Roma 15 dicembre 1944» e titolo Madrigale, prima al Gatto, è accompagnata dalla dedica a Leonor Fini (A.R.C. 52 I 4/2 (1), c. 10r), l'artista con cui la Morante, che la definisce «degna del più alto Trono degli angeliGatti-Poeti» (Morante 2012: 218), trattava principalmente di gatti, come risulta dal carteggio contenuto nell' Amata $^{29}$.

Chiude il cerchio dei personaggi felini di Alibi l'ultimo componimento, Il gatto all'uccellino (Morante 1988: 1401). In questo caso il gatto è rappresentato in confronto a un uccellino che volando gli sfugge. La poesia, con sottotitolo «Scherzo - Dedicato a $S$. P.» è dedicata a Sandro Penna, e aveva titolo originario Il gatto e l'uccellino (A.R.C. 52 I 4/1 (1), c. 9r). La sostituzione della congiunzione «e», che poneva i due animali sullo stesso livello, con la preposizione articolata, rispecchia il movimento del gatto proteso verso l'uccellino, stanziato su un piano diverso. Il punto di vista del componimento è quello del gatto che, non riuscendo a raggiungere un uccellino, «feudatario dei luoghi più alti», è costretto a fare di un «topolino di terra» la sua preda. La poesia potrebbe essere letta, secondo una lettura metaforica, come un omaggio all'amico Sandro Penna, che Elsa Morante

27 «Ed io, quasi possedessi i sensi acuti dei cani e dei gatti che, si dice, scorgono i fantasmi, avvertivo intorno i dileguanti vestigi della sua solitudine non condivisa.» (Morante 1988: 681)

${ }^{28}$ Nell'articolo Il paradiso terrestre. Il vero re degli animali è scritto: «Infelice l'uomo che ignora le consolazioni di simile amicizia! Per lui, la terra è davvero un esilio sconsolato» (Morante 1990: 1476).

${ }^{29}$ Scrive ad esempio Leonor Fini a Elsa Morante: «Mandami una poesia sui gatti - ne ò molta voglia e in questo momento scriveresti una meravigliosa poesia - spesso rileggo quella della Torre (e quella del gatto Alvaro).» (Morante 2012: 209) 
considerava straordinario poeta ${ }^{30}$. Guardando l'adorata Piazza Navona nell'articolo Navona Mia, uscito nel 1962 su Illustrazione Italiana, ora in Pro o contro la bomba atomica (Morante 1990: 1527-1536), è suo il verso che quel momento di perfezione, scandito dal «rumore dell'acqua» e dalle «voci dei ragazzini che giocano», le riporta alla mente: «Io vivere vorrei addormentato / entro il dolce rumore della vita». Continuando la riflessione, la scrittrice rimprovera ai romani di non amare abbastanza la propria piazza, definita la «principale dell'universo», e di non conoscere Sandro Penna, «il più grande poeta del mondo». I versi dell'amico sarebbero dunque di una grazia inarrivabile, come l'uccellino «pieno di grazia» si ruba al gatto, «futile minotauro negato al volo». Al di là della sua possibile interpretazione metaforica, dato importante è la figura animale con cui si chiude la raccolta, che crea un sistema con le altre due poesie feline: Minna la Siamese, la prima, è posta a una distanza di sette componimenti dal Canto per il gatto Alvaro, esattamente al centro di Alibi, mentre Il gatto all'uccellino è in chiusura dopo altri sette componimenti. La presenza del gatto dunque, non solo abbraccia l'intera raccolta, ma, trovandosi anche in posizione centrale, si conferma uno dei temi principali e dei grandi fili conduttori di Alibi.

\section{LA «CAMERA DI FAVOLE»}

Un altro grande tema di Alibi è quello della favola, intesa non come specifica delimitazione di genere, ma secondo un'accezione generica legata a una capacità immaginativa fervida e fantastica. Si è già accennato all'utilizzo del termine «alibi» in Sul romanzo per spiegare la prima persona nei romanzi. Pur appartenendo all'autrice, il protagonista ne identifica solo una porzione di realtà, creando di fatto uno sdoppiamento: «il romanziere moderno, in luogo di invocare le Muse, è indotto a suscitare un io recitante (protagonista e interprete) che gli valga da alibi». Rispetto alla comune accezione di «difesa», suggerita nell'articolo dall'autrice stessa («Quasi per significare, a propria difesa»), il sostantivo si arricchisce anche del significato etimologico di "altrove", che nella teoria dell'alibi allude alla creazione di un'identità che in relazione alla fonte è gemella e diversa, come un'immagine riflessa nello specchio. Non a caso il nucleo generativo della raccolta di Alibi, il quaderno di Narciso, era fondato sul mito del vinto dall'amore di un altro se stesso riflesso nell'acqua. I due recitanti prodotti dalla scrittrice fino al 1959, l'anno di pubblicazione dell'articolo, sono i narratori dei primi due romanzi, Menzogna e sortilegio e L'isola di Arturo. L'altrove che la Morante ha raggiunto con i suoi giovani alibi, Elisa e Arturo, è insieme spaziale e temporale: raccontare le vicende attraverso i loro occhi significa contrastare l'azione del tempo e recuperare una

${ }^{30}$ In una lettera a Giulio Einaudi la Morante scrive: «Se ti scrivo oggi, però, ho un motivo particolare: devo parlarti di un nostro comune amico, il quale è anche uno dei tuoi migliori autori, e uno dei nostri maggiori poeti: Sandro Penna.» (Morante 2012: 101) 
favola sepolta dall'età e dalla coscienza. In una lettera a Debenedetti del 18 febbraio 1957 (Morante 2012: 190-191) riguardo L'isola di Arturo, l'autrice conferma la sfumatura semantica dell'alibi come altrove spaziale: «non, però, per contraddirLa, ma solo per confessarLe che mi ha meravigliato la presenza (da Lei avvertita), in questo libro, della mia $[\mathrm{xxx}]$ intelligenza, che invece, mentre lo scrivevo, mi sembrava esclusa e lontana». Dopo aver confessato il suo "antico e inguaribile desiderio di essere un ragazzo» come ispirazione del romanzo, il significato dell'alibi si avvale dell'ulteriore valenza temporale:

Nel 1952, trovandomi, per la mia sorte, in uno stato definitivo di incompletezza e di solitudine, non vidi altra via che quella: di ritornare a $\left[\mathrm{xxx}_{\mathrm{xxx} \mathrm{xxx}]}\right.$ una mia rimpianta condizione di ragazzo, che mi sembrava di ricordare. E sempre meglio me ne ricordavo scrivendola (perciò $[\mathrm{xxx}]$ Le dicevo che mi pareva $[\mathrm{xxx}]$ necessaria quella citazione di Saba: io, se in lui mi ricordo... [xxx]). [ $\mathrm{xxx}_{\mathrm{xxx} x \mathrm{xx}]}$ Mentre scrivevo questa storia, Glielo assicuro, non mi è mai accaduto (o almeno non me ne sono mai accorta) di dovermi richiamare alla mia intelligenza, ma soltanto, davvero, a una specie di memoria.

Rinunciare all'alibi, completata la scrittura, è la cessazione di uno stato di grazia in cui la coscienza è sospesa temporaneamente grazie alla giovane età dei protagonisti. Prosegue la Morante nella lettera:

E adesso, alla fine, mi trovo peggio che dopo quell'altro mio romanzo: giacché là si è trattato, alla fine, di uscire da una camera di favole. Mentre che ora, dopo questi anni di isole e di Arturi, mi trovo ricacciata nella mia attuale e irrimediabile condizione [xxx] di donna $[\mathrm{xxx}]$ - ormai, anzi, di vecchia.

L'alibi della scrittura è dunque un incantesimo che s'illude di addomesticare il tempo, ritardando la morte. Terminato il sortilegio, l'impatto con la realtà è ogni volta più doloroso, perché la «condizione di donna ormai, anzi di vecchia» è, passando gli anni, sempre più concreta e «irrimediabile» ${ }^{31}$. Il percorso di maturazione e di presa di coscienza dei due personaggi è perciò parallelo a quello di Elsa Morante, che attraverso di loro e insieme a loro rivive il trauma della disillusione. Continua la scrittrice nella lettera a Debenedetti, a proposito di Arturo:

31 Anche nell'intervista a Schifano del 1984 la Morante connette, a proposito di Aracoeli, la scrittura alla vecchiaia, brutalmente improvvisa e ormai inesorabile. Dalle sue parole si coglie come la creazione del suo ultimo alibi Manuele, per quanto il romanzo sia «disperato», sia stata la ricerca di una «grande gioia», un tentativo di fuga dalla vecchiaia che l'ha concretamente raggiunta: «Bisogna che le dica che a sessant'anni ne dimostravo trentacinque; e poi improvvisamente, Aracoeli, che ho scritto immobile, al mio tavolo, per anni, senza mai uscire, mi ha fatto invecchiare: di colpo sono diventata vecchia. Quando ho scritto la parola fine ad Aracoeli - ed a questo proposito devo dire che non è un libro triste, come certi critici hanno scritto; disperato, forse; ma io ho provato una grande gioia mentre lo scrivevo ->. In seguito all'incitamento di Schifano, che la sprona a continuare a scrivere, risponde Elsa Morante: «Se mi rimetto a scrivere sono salva.» (Schifano 1984: 126-127) 
«Per arrivare alla maturità deve passare attraverso diversi misteri, un po' come Tamino nel Flauto Magico. Purtroppo, la vera maturità che stava ad aspettare, al di là di quei misteri, era poi quella della Sig.ra M. Ma lui, per sua fortuna, non lo sa»».

Il concetto di scrittura come creazione fantastica e illusoria, in relazione con l'inganno irreale del tempo e della morte, si esprime anche all'interno dello stesso Menzogna e sortilegio ${ }^{32}$. Rimasta prigioniera della camera delle favole ${ }^{33}$ di cui la maturità ha svelato le illusioni, l'Elisa narratrice, ormai giovane donna, è consapevole e cosciente, nel raccontare la sua storia di bambina e quella della sua famiglia, di essere una «monaca della menzogna»:

Il male velenoso della menzogna serpeggia per i rami della mia famiglia, sia paterna che materna. [...] Ma farsi adoratori e monaci della menzogna! fare di questa la propria meditazione, la propria sapienza! [...] Ecco che cosa è stata l'esistenza per me! ed ecco perché mi vedete consunta e magra al pari dei ragazzetti mangiati dalle streghe di villaggio. Essi dalle streghe, e io dalle favole, pazze e ribalde fattucchiere.

E sebbene voi dobbiate aspettarvi, o lettori, di conoscere attraverso questo libro più d'un personaggio contagiato dal nostro morbo fantastico, sappiate che il malato più grave di tutti lo avete già conosciuto. Esso non è altri se non colei che qui scrive: son io, Elisa. (Morante 1988: 22-23)

La storia della sua infanzia e della sua famiglia, rielaborata dalla mente adulta, è trasfigurata da un «morbo fantastico», la menzogna, diretta conseguenza di una capacità favolistica infantile che si scontri con la razionalità. La favola costituisce infatti per Elisa ancora bambina una via di fuga nell'inconsapevolezza ${ }^{34}$. Si pensi ad esempio a come recepisca, ancora completamente protetta, la morte della nonna Cesira: «L'ho detto, la Morte prese per me, la prima volta che m'apparve, un aspetto amabile e cerimonioso, quasi per non turbare, con tragici e squallidi aspetti, la mia mente ancora infantile» (Morante 1988: 52); la sua salma costituisce «l'ultima apparizione placida, favolosa e innocente.» (Morante 1988: 52)

Anche la scrittura di Elisa, come quella dell'autrice del libro, dispiega un sortilegio che crede di sconfiggere la morte, dando voce e vita ai fantasmi del suo

${ }^{32}$ Sull'importanza della favola in Menzogna e sortilegio, cfr. Scarano (1990).

33 «Forse, ricostruendo così tutta la nostra vicenda vera, io potrò, finalmente, gettar da un canto l'enigma dei miei anni puerili, e ogni altra familiare leggenda. Forse, costoro son tornati a me per liberarmi dalle mie streghe, le favole; attribuendo a se medesimi, e a nessun altro, la colpa d'aver fatto ammalare di menzogna la savia Elisa, voglion guarirla. Ecco perché ubbidisco alle lor voci, e scrivo: chi sa che col loro aiuto io non possa, finalmente, uscire da questa camera.» (Morante 1988: 34)

${ }^{34}$ La fascinazione ambigua della favola è costante nel romanzo. Si trova evocata ad esempio da questo pensiero di Francesco, a colloquio con Anna, che sta per sposare, diviso tra la speranza di essere ricambiato e la disillusione di ogni contraria evidenza: «E sembrò a Francesco, nel breve tempo che rimasero soli, di trovarsi in compagnia d'una favolosa Chimera, bella e feroce nel tempo stesso.» (Morante 1988: 370-371) 
passato $^{35}$ : nel sogno avuto dalla narratrice che precede la storia di Cesira, toccando il «bianco braccino» della nonna, «credendo di trovarlo ghiacciato» per via della neve che circonda la casa della sua infanzia, lo scopre invece «d'un calore vivace quasi ardente» (Morante 1988: 53). È il calore della vita che torna, nella sua favola, a scaldare i corpi costretti dal gelo della morte. Stessa operazione è condotta da Anna, che con le finte lettere di Edoardo, scambiando menzogna e verità, inventa una nuova vita per l'amato cugino.

Sono così cruciali questi due concetti, quello della finzione narrativa e della resurrezione artistica che la loro valorizzazione è affidata a due poesie, le quali, come già spiegato, lontane dall'avere un ruolo puramente esornativo, focalizzano l'attenzione sui nodi principali. Coerentemente con la sequenzialità dei due momenti, la prima, non a caso in posizione proemiale, è Alla Favola (Morante 1988: 5). La poesia è un'invocazione alla «Finzione» affinché cinga le sue sacerdotesse, Elisa ed Elsa, come una «fatua veste» ${ }^{36}$. La consapevolezza dell'artificio artistico, che si fa quasi religione, è ribadita nei versi finali «la risposta celeste / mi fingo». La seconda poesia, Ai personaggi (Morante 1988: 35), posta appena dopo la premessa, è rivolta agli attori della scena, «i Morti», i «magnifici ospiti» nella camera di Elisa, «donna sciocca e barbara», a loro «suddita e ancella». I fantasmi invocati sono i «fiori straordinari e occulti» da cui la «pronuba ape» produce il «miele» della poesia. Il racconto di Elisa è dunque un sortilegio che inganna e al contempo rimane vittima di se stesso, fondato su una favola bugiarda che volendo resuscitare i suoi fantasmi, ne sancisce di fatto l'esilio oltre un confine invalicabile. La fine del romanzo, momento fondamentale affidato nuovamente ad una poesia, il Canto per il gatto Alvaro (Morante 1988: 942-943), è per Elisa un

35 «Chi m'avesse veduta immobile per intere giornate, gli occhi spalancati e sognanti, m'avrebbe potuta credere immersa in qualche celeste meditazione; e invece, come un bevitore maniaco, io giravo nella macchinosa tregenda delle mie bugie. Bugie per qualsiasi cervello assennato; ma non per quello d'Elisa. Infatti con l'andar del tempo, io credetti nelle mie favole come in una specie di Rivelazione, e i loro personaggi non furono più, per me, delle ombre, ma quasi delle anime incarnate» (Morante 1988: 27). Anche Manuele, il protagonista di Aracoeli, ammette la scarsa credibilità del suo racconto: «"[...] Ritiene, in definitiva, il Soggetto, che le sue memorie siano ATTENDIBILI?" I. "Purtroppo, io non lo so"» (Morante 1990: 1186). Pur giudicando «apocrifi» i suoi ricordi, come le bugie di Elisa, questi risultano a Manuele «più veri del vero»: «Può darsi che questo sia uno dei miei ricordi apocrifi? Nel suo lavoro continuo, la macchina inquieta del mio cervello è capace di fabbricarmi delle ricostruzioni visionarie - a volte remote e fittizie come morgane, e a volte prossime e possessive, al punto che io m'incarno in loro. Succede, a ogni modo, che certi ricordi apocrifi dopo mi si scoprono più veri del vero» (Morante 1990: 1049-1050).

${ }^{36}$ Nell' «Introduzione» alla raccolta Garboli accosta Alla favola al sonetto di Metastasio Sogni e favole io fingo, dove pure sono centrali i temi della favola e della menzogna: «Ah che non sol quelle che io canto o scrivo / favole son: ma quanto temo o spero, / tutto è menzogna, e delirante io vivo!.» (Garboli 2004: xxi) Anche Giuseppe Leonelli, nel suo lavoro su Menzogna e sortilegio, propone un confronto tra i due componimenti (Leonelli 2012: 820-821) 
riposo temporaneo dall'istinto razionale di interrogarsi ( Si ripiega la memoria ombrosa / d'ogni domanda io voglio riposarmi»), perché il suo gatto celeste le ha fatto dono di un attimo di incoscienza, consolandola delle sue favole: «L'allegria d'averti amico / basta al cuore. E di mie fole e stragi / coi tuoi baci, coi tuoi dolci lamenti, / tu mi consoli, / o gatto mio!».

Anche nell'Isola di Arturo la favola ha un ruolo centrale e fa da spartiacque tra l'infanzia e la maturità del protagonista, nella sua ultima stagione a Procida. Preservato da un velo di Maya che gli tiene in ombra la realtà delle cose, Arturo proietta le azioni paterne in una dimensione favolistica e fantastica, che più si alimenta quanto più inaccessibile. Dopo il grande rifiuto di Wilhelm, «Sei un guaglioncello. Aspetta d'esser cresciuto, per partire con me» (Morante 1988: 990), che decreta la divisione tra Procida, terra d'infanzia, e il tanto sognato «Estero», terra della maturità, confinando Arturo a Procida, la favola delle imprese paterne ha moltiplicato la sua forza:

Così, egli lasciava la Casa dei guaglioni a passo veloce, tenendo la valigia afferrata per un capo della corda, le guance animate, gli occhi incupiti dall'impazienza: ormai già per me fiabesco e irraggiungibile, come se, gaucho, attraversasse la pampa argentina, con un toro preso al laccio; oppure, Capitano delle armate greche, trascinasse volando sul cocchio, per il campo di Troia, la spoglia del troiano vinto; o come se, domatore di cavalli nella steppa, corresse a fianco del suo puledro, pronto a saltargli in groppa nella corsa. (Morante 1988: 990-991)

Allo stesso modo la figura di sua madre, morta di parto e seppellita nel cimitero dell'isola, risente delle proiezioni favolistiche di Arturo. La «bella canaria d'oro delle favole» (Morante 1988: 1096), prima dell'arrivo di Nunziata, lo accompagna costantemente, «oziosa e contemplante», in una «tenda orientale» (Morante 1988: 1001) che ha il sapore dei racconti delle Mille e una notte, e che si nega al dominio della razionalità: «Ad ascoltare la ragione, sapevo che tutto quanto restava di mia madre era rinchiuso sotto terra, nel cimitero di Procida. Ma la ragione, davanti a lei, si ritraeva, e, senza rendermene conto, io, per lei, credevo addirittura in un paradiso» (Morante 1988: 1000). Con l'affacciarsi della coscienza, Arturo non può che rilevare la vittoria della ragione sulle sue favole. Ripiegata la tenda orientale, non è la sua assenza a pesare ad Arturo quanto la sua nuova «miscredenza»:

Perfino mia madre, la bella canaria d'oro delle favole, che, un tempo, mi veniva incontro al primo richiamo, adesso non mi soccorreva più. E la peggior cosa era questa: che non per infedeltà sua, essa mi mancava. Ero io stesso che, d'un tratto, avevo perduto ogni volontà di cercarla, negando la sua persona misteriosa. La mia miscredenza, che un tempo aveva risparmiato l'isola, adesso relegava anche lei sotto terra, fra gli altri morti che non sono più niente e non hanno nessuna risposta da dare. Seppure talvolta ero tentato dalla nostalgia di lei, subito mi dicevo crudamente: "Che ci pensi a fare! Essa è MORTA». (Morante 1988: 1096) 
Stessa disillusione, e anzi più dolorosa, riguarda Wilhelm Gerace. Dopo aver scoperto in lui la parodia dell'immagine eroica che si era costruito (Morante 1988: 1299), Arturo ripensa alla propria mitizzazione come a una «favola» tradita, «una cosa fanciullesca», d'altri tempi, e dolorosa:

Subitaneo, il ricordo della sua persona mi accorse alla mente: non come una figura precisa, ma come una specie di nube che avanza carica d'oro, azzurro torbido; o come un sapore amaro [...]. E certi tratti propri di lui, ma quasi trascurabili: una sua alzata di spalle; un suo ridere distratto; oppure la forma grande e negletta delle sue unghie; le giunture delle sue dita; o un suo ginocchio graffiato dagli scogli... ritornavano isolati, a farmi battere il cuore, quasi unici simboli perfetti di una grazia molteplice, misteriosa, senza fine... E di un dolore che mi si faceva più acerbo per questo motivo: perché sentivo che esso era una cosa fanciullesca; pari a un incontro di correnti turbinose, esso si precipitava tutto quanto in questo presente, breve passaggio d'addio! E dopo, lo avrei dimenticato, naturalmente, tradito. Di qui sarei passato a un'altra età, e avrei riguardato a lui come a una favola. (Morante 1988: 1365-1366)

Come per Menzogna e sortilegio, il senso profondo dell'Isola di Arturo è racchiuso nei versi che accompagnano il romanzo, nella poesia Dedica (Morante 1988: 947) che in Alibi prende il titolo L'isola di Arturo (Morante 1988: 1397): «Quella, che tu credevi un piccolo punto della terra, / fu tutto». La poesia è dedicata a Remo N., ormai nota abbreviazione di «Remo Natales», anagramma di Elsa Morante, com'è appuntato nelle carte del romanzo (Zagra 2008). I versi che introducono all'avventura di Arturo ne delimitano una dimensione favolosa, immatura e inviolabile («E non sarà mai rubato quest'unico tesoro / ai tuoi gelosi occhi dormienti. / Il tuo primo amore non sarà mai violato») che ha i contorni e gli spazi «di quella isoletta celeste». La maturità citata nella lettera a Debenedetti, «della Sig.ra M.», chiamata in causa dalla criptica dedica "a Remo N.», è la verità che si rivela nei versi finali e che decreta la distanza con quello che l'alibi Arturo rappresenta, una parentesi destinata a finire, come il suo stesso percorso dimostra: «E tu non saprai la legge / ch'io, come tanti, imparo, / - e a me ha spezzato il cuore: // fuori del limbo non v'è eliso».

Tenendo conto della stretta relazione tra la creazione dell'alibi e la favola, la raccolta del 1958 non poteva che contenere elementi attinenti a questa stessa sfera: le poesie di Alibi si pongono in una dimensione parallela a quella reale, nutrita dall'incanto della favola. Finzione è infatti, come già accennato, uno dei titoli presenti nel quaderno di Narciso. Il dolore della disillusione è costantemente percepibile in agguato, come per Narciso che, incontrando la proiezione irreale di se stesso, ha trovato la morte.

Il dato favolistico di Alibi è innanzitutto nelle «due fiabe per N.N.» raccolte nella sezione Allegoria, Aida e Niso. In alternativa la Morante aveva pensato, come titolo per la sezione, a «Favole per Alibi» (A.R.C. 52 I 4/1 (1), c. 27r). Nel dittico con Aida doveva inoltre esserci un componimento diverso da Niso, con titolo I due 
capretti» e sottotitolo Favola contraria a Lafontaine $^{37}$, la cui bozza è attestata tra le carte di Alibi. Si è poi già trattato del caso di Alla favola, come pure dell'utilizzo di «fole», nel Canto per il gatto Alvaro. Il termine è presente anche in Avventura (Morante 1988: 1388-1391), dove l'inseguimento dell'ennesimo dei «difficili amori» è raccontato attraverso immagini favolose, che ne rivelano tutta l'illusorietà: «Al vedermi, che per te mi consumo d'amore, / tutti mi dicono "Ah, pazza, mangiata dalle streghe, rosa dalle fole, / soldato d'imprese disperate, marinaio senza vela né remi, / dove t'avventuri? in quali deserti di sabbia, / dietro Morgane, e fuochi fatui, e larve canzonatrici / tu vuoi spegnere la tua sete nella solitaria morte!». Centrale nella raccolta è inoltre il componimento dal titolo Sheherazade (Morante 1988: 1381), la cantastorie delle Mille e una notte che con le «bellissime fiabe» è capace di «consolare la notte» vincendo la morte, perché il Sultano, il suo «sposo celeste», «padrone dei [suoi] sospiri», per ascoltarle, «benigno, ritarda per [lei] / la sentenza mortale». Il suo straordinario potere, notoriamente associato all'eternità dell'arte che neutralizza la morte, non è descritto come umano, ma come un dono del cielo: «Non è mio pregio, ma del cielo / che mi fece fantastica / se degna io sono della grazia». Il personaggio esercita un forte fascino sulla scrittrice e ha certamente contribuito a ispirare Elisa, la quale pure, in Ai personaggi, si rivolge ai fantasmi della sua storia come a dei «Sultani infingardi», gli stessi che sono riportati illusoriamente in vita dal suo racconto. In una delle finte lettere del cugino, Anna, che si è fatta cantastorie nella storia, fingendo di allontanare la morte dall'amato Edoardo, cita proprio la raccolta di fiabe: «Anna! eccomi a Costantinopoli, regno delle Mille e una notte, nido opulento di favorite e di vizir...» (Morante 1988: 791). Anche nel secondo romanzo morantiano l'atmosfera delle Mille e una notte è ben presente ${ }^{38}$, e sembra anzi essere il referente della fervida immaginazione di Arturo:

Ricordo che, in quell'epoca, facevo sogni da Mille e una notte. Sognavo di volare! Sognavo d'essere un signore magnifico, che gettava in aria alla folla migliaia di monete! $\mathrm{O}$ un gran monarca arabo, che attraversava a cavallo un deserto bruciante; e, al suo passaggio, dalle rocce del deserto sgorgavano freschissime sorgenti verso il cielo! (Morante 1988: 1242)

Elsa Morante stessa, con le sue storie e i suoi alibi, vorrebbe tardare la «sentenza mortale» rifugiandosi in un altrove favoloso ed eterno, come la Sheherazade della sua poesia, pur consapevole del disincanto inesorabile che la attende al termine della scrittura. La sera domenicale, componimento del Mondo salvato dai

${ }^{37}$ Jean de La Fontaine, scrittore francese della seconda metà del 1600, com'è noto, è autore di favole con fini moralisti.

${ }^{38}$ Si pensi ad esempio a questa considerazione di Arturo: «A volte, mi assopivo un poco sulla panca. $\mathrm{E}$ in quel sopore delicato, le minime impressioni della realtà mi si trasformavano in immaginazioni simili a frammenti d'una fiaba, che pareva volessero blandirmi infantilmente» (Morante 1988: 1146). 
ragazzini, presenta di nuovo la leggendaria cantastorie persiana, ma in una luce cupa e definitiva, che nega ogni «via di fuga» e che investe di riflesso anche la raccolta precedente: «La solita pavonessa funesta detta Sheerazade / spiega la sua ruota di trafitture, / piume e flore subito pietrificate / nella vertigine dei colori contro natura, linciaggio lacerante / di sassi puntuti. Nessuna via di fuga» (Morante 1990: 31).

Nelle intenzioni iniziali della Morante l'influenza delle Mille e una notte all'interno di Alibi avrebbe dovuto essere più vasta. Alcune carte riguardanti $\mathrm{Su}$ Nerina attestano la presenza di piccoli «racconti che mossero al pianto», definiti «favole» in un'altra scrittura della poesia (A.R.C. 52 I 4/1 (3), c. $3 r$ ), di cui non è poi rimasta traccia (A.R.C. 52 I 4/1 (1), cc. 14r-15r) ${ }^{39}$ :

"Differente da tutti. Solo un bacio d'amore

potrà vincer l'incanto." Racconti che mossero al pianto!

"L'uccello del Paradiso volò solo fra i ghiacciai del Polo

sbatté un poco le ali, e morì."

"Sulla rena secca gettato l'argenteo pesciolino

si dibatté un poco, e morì."

Ah, non dite più ${ }^{40}$, mia $^{41}$ nutrice!

non sapete che favole tristi

Dura un secolo dalla notte al giorno. Mi lasciai tentare dal sonno.

Foss'io stata Scheerazade, che seppe ingannare la morte!

In questi versi, poi non mantenuti, è piuttosto eloquente la particolare qualità attribuita e invidiata a Sheherazade, saper «ingannare la morte», diversamente dalle «favole tristi» raccontate che non hanno potere salvifico. La notte, il momento in cui la cantastorie scampa sempre al pericolo, non porta alla vita ma alla tentazione del sonno, della rinuncia e della morte. Si arricchisce allora di un nuovo significato il senso dei versi finali di Su Nerina: «Troppo lunga l'attesa del giorno. / Si lasciò tentare dal sonno» (Morante 1988: 1396). Anche Alibi, poesia centrale della raccolta, di cui è non a caso omonima, conteneva in fase manoscritta allusioni dirette alle Mille e una notte. Il riferimento del verso «Tu eri il paggio favorito alla corte d'Oriente» era più esplicito nella sua versione precedente, espunta, «Tu eri l'alunno favorito del Vizir» (A.R.C. 52 I 4/1 (3), c. 17r), specificata dall'appunto autografo, di un'altra carta, «Scheherazade la figlia del Vizir» (A.R.C. 52 I 4/1 (3), c. $9 r$ ). Altri versi conservati in varie carte ne dimostrano ulteriormente l'importanza: «Tu sei mille destini, sei tutte le fiabe d'Arabia» (espunto) (A.R.C. 52 I 4/1 (3), c.

\footnotetext{
${ }^{39}$ Le trascrizioni dalle carte non riportano per intero le numerose cancellature, ma solo il testo secondo l'ultima versione. Sono segnalate in nota le varianti non espunte dall'autrice.

${ }_{40}^{40}$ «non dite più» è sovrascritto al testo espunto («non parlate più») tra parentesi quadre, mentre «[tacete]», è sottoscritto a «[non parlate più]», a sua volta sottoscritto al testo espunto.

${ }^{41}$ «mia» è inserito in interlinea superiore tra parentesi quadre.
} 
64r), «Le tue mille storie conosco. Un'altra ne vuoi sapere?» (A.R.C. 52 I 4/1 (3), c. $9 r$ ). Di queste prove rimane traccia, anche se meno evidente, nel verso definitivo «Tu sei la fiaba estrema», in cui l'aggettivo, in relazione con la «fiaba», riporta alla mente il verso leopardiano di $A$ se stesso che decreta la fine dell'ultima disperata illusione: «Perì l'inganno estremo». La «fiaba estrema» raccontata in Alibi torna, con i medesimi termini, nelle considerazioni di Manuele, protagonista di Aracoeli e ultimo alibi di Elsa Morante:

Ciascun viaggiatore del veicolo è escluso dall'altro, e così io da tutti. E come succede a certi monaci oranti nei mercati, meravigliosamente il baccano estraneo, sbarrando ai miei sensi le voci prossime e facendomi, anzi, da scudo, mi ha ristretto nella clausura appartata della mia fiaba estrema. (Morante 1990: 1198)

La sua «fiaba estrema», lo «scudo» di Manuele in viaggio verso El Almendral, è ciò che rimane della facoltà, posseduta da bambino, di ornare il reale di favolose sfumature. Esotica e misteriosa, nella sua beata «ignoranza», è la visione ch'egli ha di Aracoeli:

Però sempre, in quei pianeti inesplorati della mia ignoranza, mia madre poteva discendere da una stirpe di gitani o di mendicanti o di toreri o di banditi o di Grandi Idalghi (forse, avrà avuto dimora in un Castello inaccessibile? Forse in qualche alhambra o alcázar?). (Morante 1990: 1068)

Il percorso di Manuele in terra di Spagna, speculare e contrario a quello dell'Isola di Arturo, nella sua doppia direzione dello spazio e del tempo, è un tentativo estremo di ritrovare rifugio in una dimensione illusoria e perduta, protetta dall'abbraccio materno. Il viaggio alla ricerca di Aracoeli è contemporaneamente la ricerca disperata della «favola di Totetaco» (Morante 1990: 1262) (2) $^{42}$ e dell'inconsapevolezza dell'infanzia. Come per i primi romanzi, anche l'ultimo racconto andaluso risente dell'influenza di Sheherazade e delle Mille e una notte. Il libro è citato nella descrizione della Porta di Almeria, città andalusa e per questo fiabesca che «in arabo significa specchio» ${ }^{43}$ (Morante 1990: 1087), ritratta in una delle tre cartoline ammirate dal ragazzetto Manuele:

La prima è un'antica veduta della Porta di Almeria (la puerta de oro) disegno da Mille e una notte, stampato in un colore ocra solare (l'oro) con qualche macchia d'azzurro e di vermiglio. Simile a un'altissima nave, questa Porta si erge a specchio di un approdo popolato di vele e di minuscoli personaggi d'oriente. (Morante 1990: 1087-1088)

42 «Totetaco» è il quartiere romano di Montesacro così pronunciato da Manuele bambino, dove egli ha trascorso con i suoi genitori, ma soprattutto, con Aracoeli, una felice e mai dimenticata infanzia.

${ }^{43} \mathrm{Si}$ pensi a quanto accennato riguardo l'importanza del mito di Narciso nella teoria dell'alibi e nello scontro con la finzione. 
Persino l'entrata di un bordello ha per il fanciullo Manuele, la cui percezione è filtrata dallo schermo della favola, il fascino delle Mille e una notte: «Quel cancello, marchiato da interdizione e maledetto, rimase, per me bambino, l'ingresso al Palazzo delle Mille e una Notte.» (Morante 1990: 1117)

Cantastorie e spesso uditrice delle fiabe di Manuele ${ }^{44}$ nell'idillio felice a Montesacro, è proprio Aracoeli, nuova e ultima rappresentazione di Sheherazade. L'incanto della favola è anche in questo frangente vincolato dalla «maturità». Sempre più lontana dalla sua primitiva e barbara ingenuità dopo essere stata trapiantata alla casa dei Quartieri Alti, Aracoeli, un tempo «cantastorie eccelsa»e curiosa ascoltatrice, spinta dal desiderio di essere «degna del rango» di suo marito, ignora $\mathrm{i}$ «soggetti fiabeschi» di suo figlio, «non più adatti alla sua maturità», per «riviste di moda»e «settimanali illustrati»:

Ma anche qui (lei, già stata cantastorie eccelsa, e poi compagna delle nostre fiabesche letture serali!) mia madre adesso pareva immettersi a ritroso, altalenando fra una certa nostalgia di giochi e un'opportuna rinuncia. Indi la rinuncia fatalmente prevalse; e finì che lei, da se stessa, si espulse da quel nostro piccolo feudo comune. Mentre, ansioso di dialogo, io m'infervoravo nei miei soggetti fiabeschi, essa ormai, disincantata e distratta, mi badava a malapena e con una certa aria d'indulgenza superiore: quasi alienandosi di proposito da simili interessi per lei datati, e non più adatti alla sua maturità. Attualmente, per lei, maturità significava: perizia e costumanze da signora, degna del rango di mio padre. E a tale esigenza ingenua, le rispondevano meglio le riviste di moda e $\mathrm{i}$ settimanali illustrati, con le foto delle indossatrici dei regnanti e dei divi. Essa li esaminava con la diligenza accigliata e un poco forzosa di un piccolo zelante ebreo chino sul Talmud o sulla Torah. E brontolava se a me talvolta capitava d'interromperla, respingendomi addirittura, al modo che un filosofo scansa un caro gattino venuto a scompigliare le sue carte. (Morante 1990: 1263)

Nell'eloquente similitudine finale, nell'atto di coinvolgere Aracoeli nelle sue storie fiabesche, Manuele è come «un caro gattino», emblema d'innocente inconsapevolezza, che scompiglia le carte di un filosofo, simbolo della coscienza razionale, emersa con la maturità, che rinnega l'incoscienza felice ma caduca delle favole.

${ }^{44}$ «Certe volte, nel vedermi intento alla lettura di qualche favola, essa (come già usava al tempo antico) mi diceva: "è bella? me la conti?" protendendosi curiosa» (Morante 1990: 1304). 


\section{RIFERIMENTI BIBLIOGRAFICI}

BARBATO, Andrea (1963): «Attraverso occhi adolescenti riesce a individuare la realtà», Il Giorno, 4 settembre 1963, p. 3.

BARDINI, Marco (1999): Morante Elsa. Italiana. Di professione, poeta, Pisa, NistriLischi.

CAPRONI, Giorgio (1958): «La grande pioggia», La Fiera Letteraria, 27 luglio, p. 3. CECCHI, Carlo; GARBOLI, Cesare (1988): Cronologia, in Elsa Morante: Opere, vol. 1, Milano, Mondadori, pp. XVII-XC.

Costantini, Costanzo (1980): «Vorrei essere un fantasma», Il Messaggero, 13 gennaio 1980, p. 3.

GARBOLI, Cesare (2004): «Introduzione», in: Elsa Morante: Alibi. In appendice: Quaderno inedito di Narciso, Torino, Einaudi, pp. vii-xxii.

DEL FRA, Lino (1957): «Elsa Morante Premio Strega», L'Italia che scrive, a. 40, n. 7-8 (lug.-ago. 1957), p. 135.

FIORILLA, Maurizio (2009): «Tra le carte del "Mondo salvato dai ragazzini" di Elsa Morante: per la genesi di "Addio"», in Simona Brambilla / Maurizio Fiorilla (a cura di), La filologia dei testi d'autore. Atti del Seminario di Studi (Università degli Studi Roma Tre, 3-4 ottobre 2007), Firenze, Cesati, pp. 243-268.

LEONELLI, Giuseppe (2012):«"Finzione...fatua veste”. Lettura di Menzogna $e$ sortilegio», Studium, nov./dic. 2012 (anno 108), n. 6, numero a cura di Giuseppe Leonelli, Elsa Morante. Nel centenario della nascita, pp. 810-826.

MASSARI, Giulia (1957): «L'isola di Elsa», Il Mondo, 19 marzo 1957, p. 15.

MORANTE, Daniele (2012) (a cura di): L'Amata. Lettere di e a Elsa Morante, con la collaborazione di Giuliana Zagra, Torino, Einaudi.

MORANTE, Elsa (1958): Alibi, Milano, Longanesi.

MORANTE, Elsa (1988): Opere, a cura di Carlo Cecchi e Cesare Garboli, vol.1, Milano, Mondadori.

MORANTE, Elsa (1990): Opere, a cura di Carlo Cecchi e Cesare Garboli, vol. 2, Milano, Mondadori.

PogGio, Piero (1984): «Non mi piace essere fotografata, però devo ammetterlo: sono ancora carina», Gente, a. 28, n. 50 (14 dicembre 1984), pp. 34-38.

PORCIANI, Elena (2006): L'alibi del sogno nella scrittura giovanile di Elsa Morante, Soveria Mannelli, Iride Edizioni.

RosA, Giovanna (1995): Cattedrali di carta. Elsa Morante romanziere, Milano, il Saggiatore.

SAVIANE, Sergio (1955): «Elsa Morante e L'Isola di Arturo», L'Espresso, a. 1, n. 1 (2 ott. 1955), p. 11.

SCARANO, Emanuella (1990): «La "fatua veste" del vero», in Lucio Lugnani et alii (a cura di), Per Elisa. Studi su Menzogna e sortilegio, Pisa, Nistri-Lischi, pp. 95-171.

SCHIFANO, Jean-Noël (1984): «Barbara e divina», L'Espresso, 2 dicembre 1984, pp. 122-133.

VENTURI, Gianni (1977): Elsa Morante, Firenze, La Nuova Italia. 
ZAgRA, Giuliana; ButTò, Simonetta (a cura di) (2006): Le stanze di Elsa. Dentro la scrittura di Elsa Morante, Roma, Colombo.

ZAGRA, Giuliana (2008): «I nomi nascosti nella dedica de L'isola di Arturo», L'Ellisse, 3, pp. 153-160.

ZAGRA, Giuliana (a cura di) (2012): Santi, Sultani e Gran Capitani in camera mia. Inediti e ritrovati dall'Archivio di Elsa Morante, Roma, Biblioteca Nazionale Centrale. 